\title{
Design and Analysis of Low Emissivity Radiative Cooling Multilayer Films Based on Effective Medium Theory
}

\author{
Haiyan Yu, Haochun Zhang, Zanen Dai and Xinlin Xia
}

Radiative cooling films are energy-efficient devices widely used in several domestic and industrial applications. To broaden its application in systems that require higher temperature requirements, this study designed low emissivity multilayer films using effective medium theory. The multilayer films obtained reflectivity greater than 0.99 at temperature regions of 207-362 K, 580-960 K and 1159-1932 K. Combinations of these basic films resulted in the bottom layer being significantly impacted upon during cooling radiation. Furthermore, the radiative cooling films with 6 periods, high refractive index ratio, and $0.014 \mu \mathrm{m}$ root mean squared roughness achieved the optimal application effect and economic benefits.

Keywords: Cooling radiation; Multilayer films; Effective medium theory

Received 19 August 2019, Accepted 13 October 2019

DOI: $10.30919 /$ esee8c333

\section{Introduction}

Total energy consumption and consequent greenhouse effect have increased dramatically in recent decades. In solving this increasingly serious problem, scholars have proposed metamaterials, ${ }^{1,2}$ nanofluid, ${ }^{3-5}$ radiative cooling radiators, ${ }^{6,7}$ solar cell, ${ }^{8,9}$ etc. from three basic heat transfer ways. As an effective strategy to radiating the heat into the outer atmosphere, multilayers energy-efficient radiators, is widely used in building materials, ${ }^{10}$ food transportation, ${ }^{11}$ electronic components, ${ }^{12}$ etc. due to their light weight, low cost and easy to process. Paul ${ }^{13}$ proposed a lithium fluoride $(\mathrm{LiF})$ and magnesium oxide $(\mathrm{MgO})$ multilayer positive cooling radiator which achieved over $85 \mathrm{~W} \cdot \mathrm{m}^{-2}$ net radiative power at clear night sky. Gentle et al. ${ }^{14}$ established a silver and polymer multilayer film which attained approximately $100 \%$ reflectance at the range of $7.9-13 \mu \mathrm{m}$ wavelength. Huang et al. ${ }^{15}$ proposed an invisible radiative cooling coat consisting of a random-stacked multilayer of calcium fluoride $\left(\mathrm{CaF}_{2}\right)$ and germanium $(\mathrm{Ge})$ which served a high reflectance in the wavelength of 3-5 $\mu \mathrm{m}$ and $8-12 \mu \mathrm{m}$ while simultaneously achieving a high emissivity in 5-8 $\mu \mathrm{m}$. Notably, $\mathrm{SiO}_{2}$ is a special and preeminent material of interest for radiative cooling. ${ }^{16}$ Granqvist et $a l^{17}$ produced a $\mathrm{SiO}_{2}$ and hafnium dioxide multilayer radiator with $\mathrm{Ag}$ top and silicon substrates. They found that the optimum cooling performance, means that $14{ }^{\circ} \mathrm{C}$ below ambient surrounding, happened at the $1 \mu \mathrm{m}$ thickness. Kecebas et al. ${ }^{18}$ designed a $\mathrm{SiO}_{2}$ and $\mathrm{TiO}_{2}$ multilayer film with aluminum dioxide $\left(\mathrm{Al}_{2} \mathrm{O}_{3}\right)$ topped, which increased thermal emission and solar reflection at $10 \mu \mathrm{m}$ incident wavelength.

Effective Medium Theory (EMT), as a typical numerical

School of Energy Science and Engineering, Harbin Institute of Technology, Harbin 150001, China

*E-mail: hczhang@hit.edu.cn calculation method, ${ }^{19,20}$ is widely used to scientifically solve the equivalent medium equations for different materials. Bates back to 1837, Faraday proposed one of the earliest models for a composite metal-insulator dielectric. ${ }^{21}$ Maxwell around 1870, and Garnett in 1904 developed models to describe this composite which is known as Maxwell-Garnett theory. ${ }^{22}$ At the field of micro scale radiation, further researches have been doneto find ways to realize energy regulation using the equivalent medium theory. ${ }^{23-25}$ Wang et al. ${ }^{26}$ studied the anisotropic optical properties of silicon solar thin-film with equivalent medium theory. Liu et al. ${ }^{27,}{ }^{28}$ predicted the near field radiative heat transfer using the equivalent medium theory on multi-film metamaterials, which was presented quantitatively. They also used this method to analyze the near-field radiation of metasurfaces. Zhao et al. ${ }^{29}, 30$ applied this theory to the thermal emission control of magnetic polaritons in 1D and 2D tungsten grating emitters. They predicted the effective hyperbolic regions using the EMT between graphene and hexagonal boron nitride multilayers. Zhang et $a l .^{31}$ examined the applicability of the medium-homogenized equivalent medium theory in place of multilayered thin-film optics. In terms of structural design, Zhang et al..$^{32}$ proposed EMT for anisotropic metamaterials, which broadened the applicability of this theory thereby providing more possibilities in anisotropic material designing. Petersen et $a .^{33}$ offered an improved EMT in graphite and hexagonal boron nitride multilayers metamaterials, which presented improved convergence in the lowenergy regime calculated by transfer matrix method. Huang et $a l^{34}$ studied a wideband metamaterial absorber and analyzed its internal mechanism using the equivalent medium theory. Li et al..$^{35}$ provided equivalent medium theory for functional graded particle with anisotropic shells. Additionally, they transformed the heterogeneous and anisotropic particle into a homogeneous, continuous and isotropic sphere.

Scholars have mainly focused on cooling radiation applications at room temperature. However, in the high-temperature working 
environment such as industrial boilers ${ }^{19}$ and electronic detector, ${ }^{36}$ active cooling is mainly used at the expense of electric energy or other energy sources. Here, we use a multilayer membrane structure to explore passive cooling in high temperature environments at the near-infrared and mid-infrared atmospheric windows, to ensure the best results and the smallest energy. Therefore, in this study, a multilayer radiative cooling device was used to investigate three atmospheric windows, ${ }^{36,37}$ near infrared $(1.5-2.5 \mu \mathrm{m})$, medium infrared $(3-5 \mu \mathrm{m})$ and far infrared $(8-14 \mu \mathrm{m})$. This was aimed at using multifilm structures' reflectivity to calculate the effects of radiation cooling on low emissivity materials namely; $\mathrm{PbS}$ and $\mathrm{SiO}_{2}$. In addition, the effect of period number $\left(N_{\mathrm{c}}\right)$, stacking modes, material properties, as well as the roughness surface, which is inevitable during manufacturing processing, were considered in the multi-films radiant cooling device. This research which is based on transfer matrix method provides a new approach in designing and analysing the mechanism of thermal reflective insulation multilayers.

\section{Experimental section}

\subsection{Mathematical model}

Transfer matrix method (TMM) also called characteristic matrix method or transmission matrix method, mainly expresses the information of the electric field and magnetic field on the surface of the incident and emergent dielectric layers in the form of a matrix. The transmission and reflection of the structure through the characteristic matrix of the electric and magnetic field, can be obtained by TMM through a series of conversion.

Fig. 1 shows a schematic diagram of an $i$-layer film. The media numbers on both sides of this structure are 0 and $i+1$. When a beam of light $\lambda$ with wavelength is incident on the multilayer films, the transmission matrix of the medium is as follows ${ }^{38,39}$ :

$$
M_{i}=\left[\begin{array}{cc}
\cos \delta_{i} & \frac{j}{n_{i}} \sin \delta_{i} \\
j n_{i} \sin \delta_{i} & \cos \delta_{i}
\end{array}\right]
$$

where $\delta_{\mathrm{i}=} k n_{i} d_{i}$, is the effective phase shift thickness of the film layer, $d_{i}$ is the thickness of this layer, $n$ is the refractive index, and the wave vector $k=2 \pi / \lambda$. The transfer matrix of the multilayer membrane structure is:

$$
M=\prod_{i=1}^{t} M_{i}=\left(\begin{array}{ll}
m_{11} & m_{12} \\
m_{21} & m_{22}
\end{array}\right)
$$

Therefore, the electric and magnetic fields on both sides of the multilayer membrane structure can be calculated in equation (3):

$$
\left[\begin{array}{l}
E_{0} \\
H_{0}
\end{array}\right]=M_{1} \cdot M_{2} \cdot M_{3} \cdots M_{t}=\left(\begin{array}{ll}
m_{11} & m_{12} \\
m_{21} & m_{22}
\end{array}\right)\left[\begin{array}{l}
E_{t+1} \\
H_{t+1}
\end{array}\right]
$$

where $E_{0}, H_{0}, E_{\mathrm{t}+1}, H_{\mathrm{t}+1}$, respectively, represent electromagnetic fields at the outer surface of the multilayer films. When the electromagnetic wave is incident vertically, the reflection coefficient $r$ and transmission coefficient $t$ of the multilayer film structure are:

$$
\begin{aligned}
& r=\frac{\left(m_{11}+m_{12} n_{t+1}\right) n_{0}-\left(m_{21}+m_{22} n_{t+1}\right)}{\left(m_{11}+m_{12} n_{t+1}\right) n_{0}+\left(m_{21}+m_{22} n_{t+1}\right)} \\
& t=\frac{2 n_{0}}{\left(m_{11}+m_{12} n_{t+1}\right) n_{0}+\left(m_{21}+m_{22} n_{t+1}\right)}
\end{aligned}
$$

The reflectance $R$ and transmittance $T$ of that material can be further obtained from the reflectance and transmittance calculated according to equation (4) and equation (5). The calculation formula can then be expressed as follows:

$$
R=|r|^{2}, \quad T=\frac{n_{t+1}}{n_{0}}|t|^{2}
$$

The magnetic fields $H^{\prime}$ and $H$ in the transmission matrix are then normalized by $H_{r e d}^{\prime}=\left(\mathrm{i} \omega \mu_{0}\right) H^{\prime}$ and $H_{r d}=\left(\mathrm{i} \omega \mu_{0}\right) H$, respectively, and the transmission matrix can be converted into:

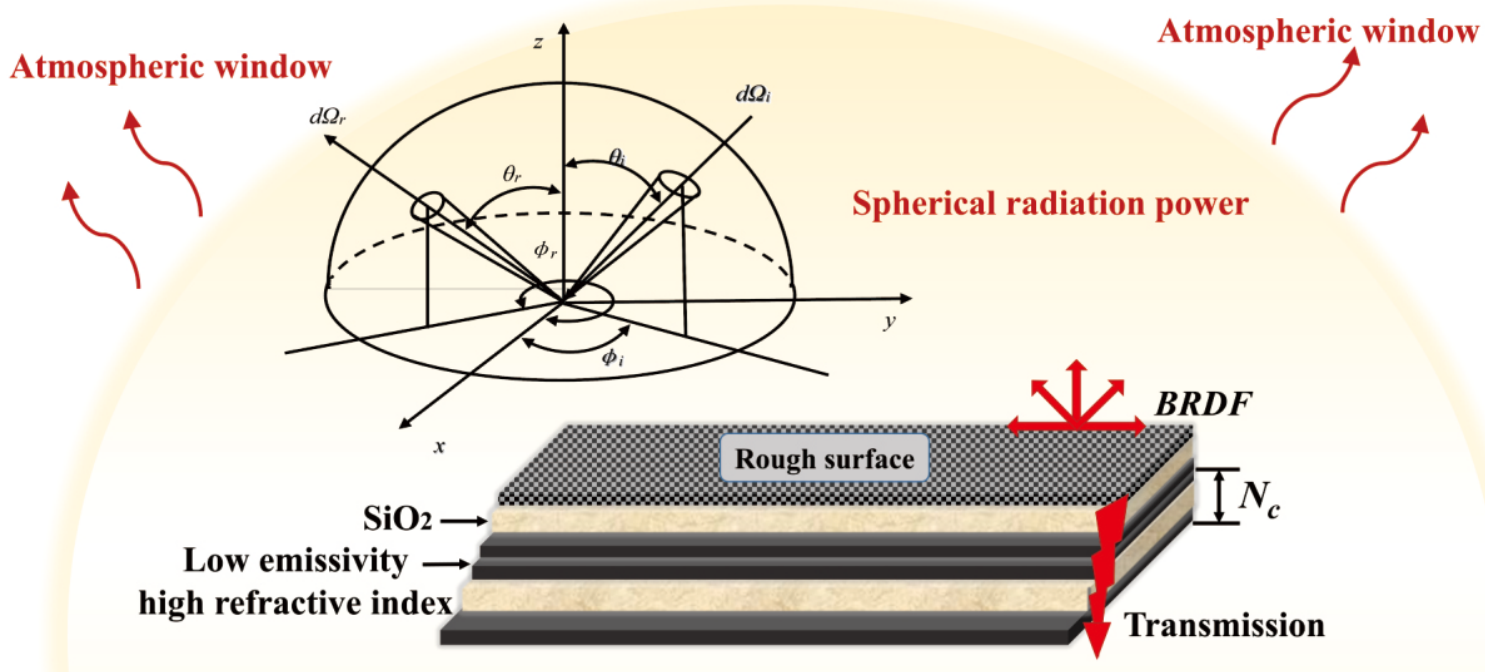

Fig. 1 Schematic diagram of multilayer films. 


$$
T_{i}=\left[\begin{array}{cc}
\cos \delta_{i} & \frac{Z_{i}}{k} \sin \delta_{i} \\
\frac{k}{Z_{i}} \sin \delta_{i} & \cos \delta_{i}
\end{array}\right]
$$

where $Z_{\mathrm{i}}$ is the wave impedance of the $i$-th layer medium, the formula (3) can be changed to:

$$
\left[\begin{array}{c}
E_{0}^{\prime} \\
H_{0, \text { red }}^{\prime}
\end{array}\right]=T_{1} \cdot T_{2} \cdot T_{3} \cdots T_{t}=\left(\begin{array}{cc}
T_{11} & T_{12} \\
T_{21} & T_{22}
\end{array}\right)\left[\begin{array}{c}
E_{t+1} \\
H_{t+1, \text { red }}
\end{array}\right]
$$

Regarding FP resonance, when electromagnetic waves are incident, multiple reflections occur on the surface of each film layer, and when the phase difference between the two reflected waves is $\pi$, destructive interference occur. If the reflected light at the next boundary of a certain film is $\pi(1+2 x)$, this will lead to destructive interference, according to equivalent medium effective medium theory which is:

$$
n \cdot d=(1+2 x) \lambda_{x} / 4
$$

where $x$ is the positive integer, $\lambda_{x}$ incident wave. Pertaining to roughness of surfaces of multilayers due to processing activity, the reflectivity $R$ and Bidirectional Reflectance Distribution Function $(B R D F)$ can be discussed using the following equations: $:^{23}$

$$
R=\frac{P_{r}}{P_{i}}
$$

$$
\operatorname{BRDF}\left(\theta_{r}, \varphi_{r}\right)=\frac{d P_{\mathrm{r}}\left(\theta_{r}, \varphi_{r}\right)}{P_{i}\left(\theta_{i}, \varphi_{i}\right) \cos \left(\theta_{r}\right) d \Omega_{r}\left(\theta_{r}, \varphi_{r}\right)}
$$

where $P_{r}$ is the power of reflected light, $P_{i}$ is the power of incident light, $\theta_{i}, \varphi_{\mathrm{i}}$ are the zenith angle and azimuth angle of incident light, and the $\theta_{n}$ $\varphi_{\mathrm{r}}$ denote the zenith angle and azimuth angle of reflected light, respectively, $d \Omega_{i}$ and $d \Omega_{r}$ are the detector solid angles of incident light and reflected light, respectively.

\subsection{Physical model}

In this study, the radiation characteristics of multilayer films at near infrared (1.5-2.5 $\mu \mathrm{m})$, medium infrared (3-5 $\mu \mathrm{m})$ and far infrared (8-4 $\mu \mathrm{m})$ windows were calculated respectively by using the transfer matrix method. According to Kirchhoff's law, ${ }^{40}$ the absorptivity of object is numerically equal to its emissivity, which is the physical property parameter of the material. Therefore, this present study focused on the reflectivity of multilayer film to calculate the effects of radiation. The detailed design process of the radiative cooling multilayer films in this present research is shown in Fig. 2.

Low emissivity materials $\mathrm{PbS}$ and $\mathrm{SiO}_{2}$ were selected, with refractive indexes of 4.1 and 1.45 , respectively. These two media have no magnetism in infrared band and their permeability is 1 . The dielectric materials on both sides of the multilayer film are air, with its refractive index, the dielectric constant and permeability all equal to 1 . The central wavelength of the three atmosphere windows were; $2 \mu \mathrm{m}, 4$ $\mu \mathrm{m}$ and $10.6 \mu \mathrm{m}$, respectively. According to equation (9), the thicknesses of the multilayer films were calculated satisfying the interference conditions, as shown in Table 1.

Table 1 Film materials and thicknesses corresponding to different center wavelengths.

\begin{tabular}{ccccc}
\hline Material & Refractive index & $\begin{array}{c}d_{1} / \mu \mathrm{m} \\
\lambda_{0}=2 \mu \mathrm{m}\end{array}$ & $\begin{array}{c}d_{2} / \mu \mathrm{m} \\
\lambda_{0}=4 \mu \mathrm{m}\end{array}$ & $\begin{array}{c}d_{3} / \mu \mathrm{m} \\
\lambda_{0}=10.6 \mu \mathrm{m}\end{array}$ \\
\hline $\mathrm{PbS}$ & 4.1 & 0.122 & 0.244 & 0.646 \\
$\mathrm{SiO}_{2}$ & 1.45 & 0.345 & 0.690 & 1.828 \\
\hline
\end{tabular}

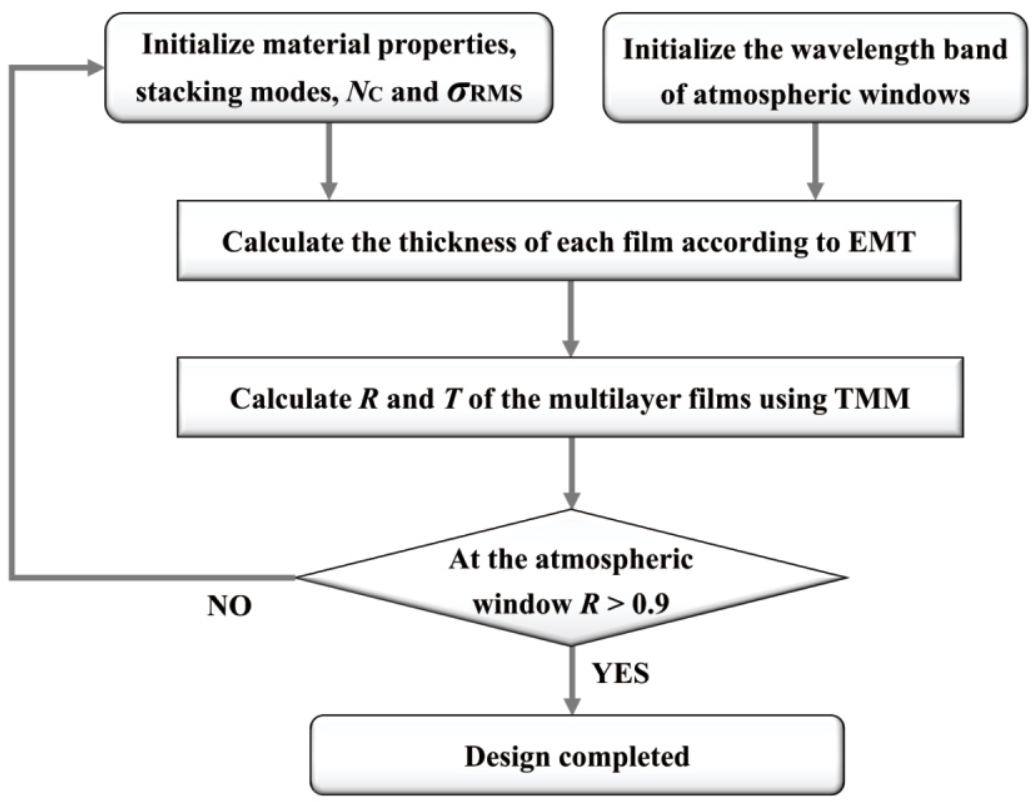

Fig. 2 Flow diagram of radiative cooling multilayer films design. 
In order to verify the independence of calculation models, the radiation characteristics of a six-layer $\mathrm{PbS} / \mathrm{SiO}_{2}$ film with a central wavelength of $4 \mu \mathrm{m}$ interference was calculated by using transfer matrix method (TMM) and finite difference time domain method (FDTD), respectively, with the range of incident wavelength from 2.5 $\mu \mathrm{m}$ to $14 \mu \mathrm{m}$. The calculation results are shown in Fig 3 .

It can be seen from the Fig. 3, that the results of reflectance and transmittance curves were almost completely coincident by these two calculation methods. Therefore, it can be verified that the correct calculation result can be obtained by using the transfer matrix method.
Compared with FDTD method, TMM approach applied to the multilayers is more accurate for the spectrum predication, without the iteration error caused by complex internal boundaries. Although the FDTD method can be applied to a more complex structure, multilayer film structure is more convenient and accurate by using the TMM in our next numerical simulation works.

\section{Results and discussion}

3.1 Multilayer films for each three atmospheric windows

The reflectivity of $\mathrm{SiO}_{2} / \mathrm{PbS}$ six-priodic films at three atmospheric
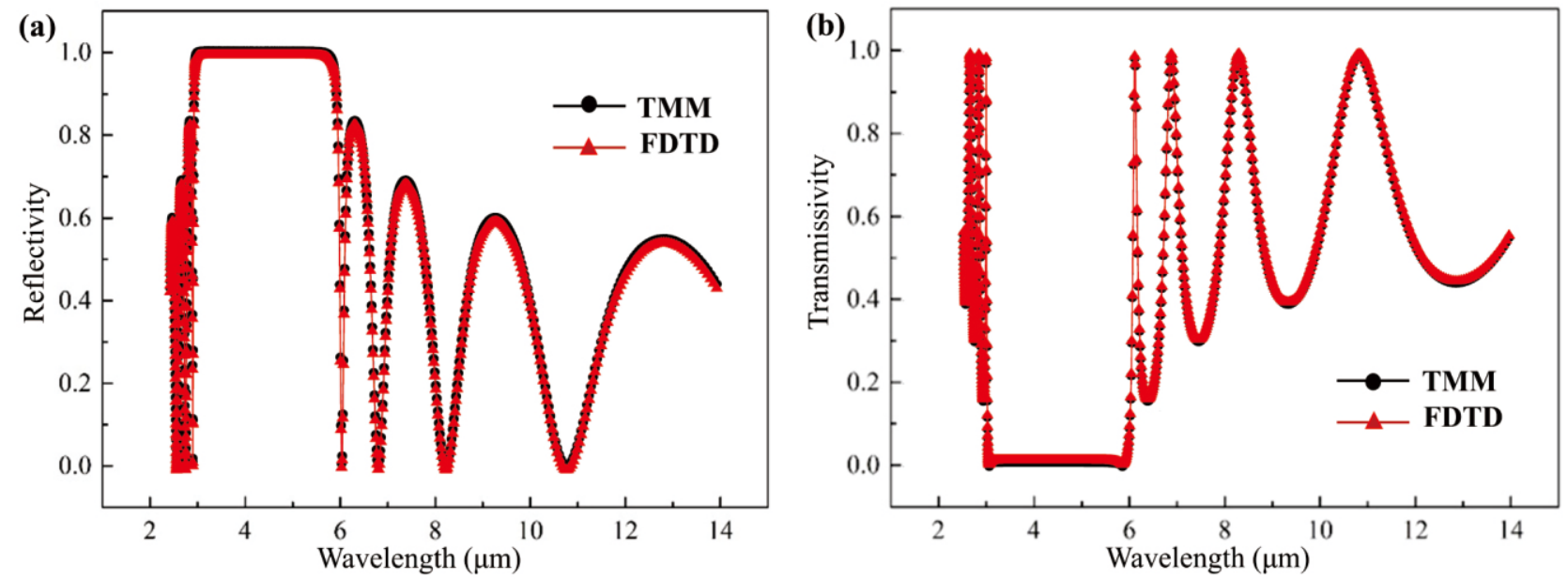

Fig. 3 Validation of numerical calculation method: (a) the reflectivity; (b) the transmissivity.
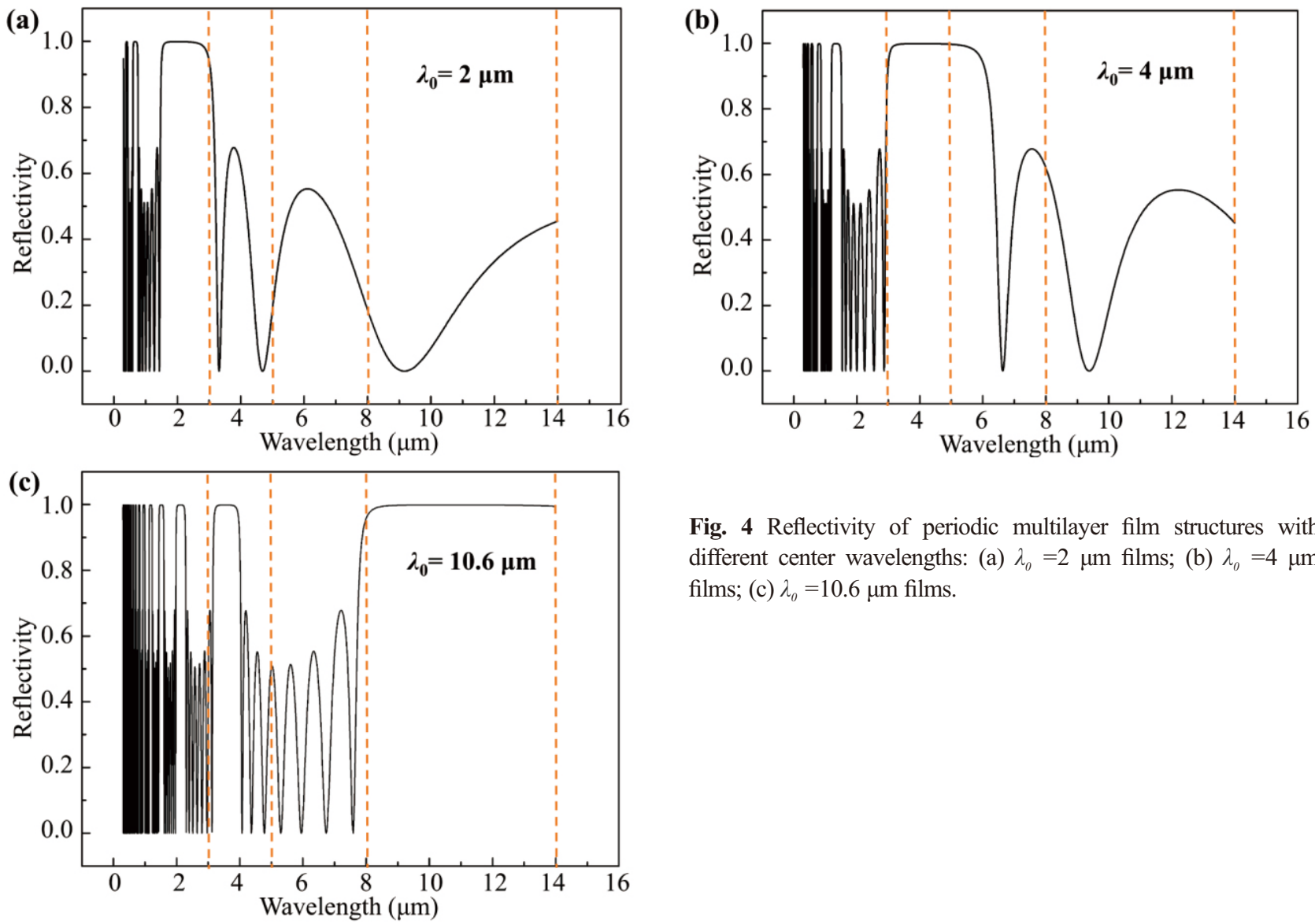

Fig. 4 Reflectivity of periodic multilayer film structures with different center wavelengths: (a) $\lambda_{0}=2 \mu \mathrm{m}$ films; (b) $\lambda_{0}=4 \mu \mathrm{m}$ films; (c) $\lambda_{0}=10.6 \mu \mathrm{m}$ films. 
windows were calculated by using the transfer matrix method (Fig. 4). The $2 \mu \mathrm{m}$ - central -wavelength $\left(\lambda_{0}=2 \mu \mathrm{m}\right)$ films had a reflectivity which was more than 0.99 , at the range of wavelength from $1.50-2.98 \mu \mathrm{m}$ (Fig. 4a). In the infrared band of 1.48-3.06 $\mu \mathrm{m}$, the reflectivity of the films was greater than 0.95 . This suggests that these films generated a positive effect on cooling radiation at high temperatures of $947-1950 \mathrm{~K}$ displaced by Wien's law. ${ }^{40}$

The $\lambda_{0}=4 \mu \mathrm{m}$ films had greater than 0.99 reflectivity at the range of wavelength from 3-5.96 $\mu \mathrm{m}$ (Fig. 4b). At infrared bands within 2.97$6.12 \mu \mathrm{m}$, the reflectivity of this films was larger than 0.95 , which content the requirements of cooling radiation in the temperature of 473$975 \mathrm{~K} . \lambda_{0}=10.6 \mu \mathrm{m}$ films had a reflectivity greater than 0.99 , at $7.97-14$ $\mu \mathrm{m}$ far infrared wavelength range (Fig. 4c). Thus, reflectivity of these films were lager than 0.95 at wavelength of 7.88-14.00 $\mu \mathrm{m}$, which signifies a favorable radiative cooling capability in the temperature interval of 207-363 K.

Therefore, the three central wavelength multilayer films designed for the three atmospheric windows achieved reflection-based radiation cooling in the high, medium and low temperature sections, respectively. Consequently, these three basic structures will be used to study the influencing factors of their radiation effects.

\subsection{Influencing factors \\ Stacking modes}

The radiant cooling effect of films in a single temperature range was studied in the previous section. However, when the working environment required a wide temperature range or a variable temperature environment, the three $\mathrm{SiO}_{2} / \mathrm{PbS}$ multilayer films were stacked to meet the actual requirements. Thus, this section will focus on the effect of the stacking modes on their cooling radiation. Each basic multilayer film consisted of four periods with central wavelengths of $2 \mu \mathrm{m}, 4 \mu \mathrm{m}$ and $10.6 \mu \mathrm{m}$ respectively. The calculation models was named according to the order of stacking modes. That is, $2-4 \mu \mathrm{m}$ means that the uppermost layer was a basic $\lambda_{0}=2 \mu \mathrm{m}$ film, followed by the basic $\lambda_{0}=4 \mu \mathrm{m}$ film, along the direction of wave propagation, and so on. The calculation results of two stacked structures are shown in Fig. 5.

The optimum reflection region of the $4-2 \mu \mathrm{m}$ structure was the 1.5-2.5 $\mu \mathrm{m}$ wavelength band, while the optimum reflection region of the 2-4 $\mu \mathrm{m}$ structure occurred at 3-5 $\mu \mathrm{m}$ wavelength range, where the reflectivity was greater than 0.95 (Fig. 5a). Similarly, as shown in Fig. 5 (b) and Fig. 5 (c), the optimal reflection area of the entire structure had a reflectivity greater than 0.95 , which corresponded to the optimal reflection area of the bottom layer of the basic film too. This phenomenon may have occurred due to the modulation of electromagnetic waves at the last film after passing through several layers of the structure. That is, the film at the bottom corresponded to a layer of filter which retained the radiation characteristics of the last film. Therefore, the overall reflection control characteristics of these combined structures were most affected by the bottom film. To fully verify this conclusion, we calculated the structure of the three-film stacked structure in the range of $0.5-14 \mu \mathrm{m}$, with the results shown in Fig. 6.

The structure was stacked in the order of the central wavelength from 2-4-10.6 $\mu \mathrm{m}$, and the transmittance and reflectance of the radiation cooling structure were obtained and presented in Fig. 6 . The reflectivity at three atmospheric windows were all close to 1 , and the transmittance were close to zero. When the wavelength at the range of $6 \mu \mathrm{m}-8 \mu \mathrm{m}$ was less than $1.1 \mu \mathrm{m}$, the curve changed drastically due to the concussive emissivity of the material, where the energy absorbed by the structure was emitted. According to this principle, design of such structures can be achieved in order to satisfy the different requirements of high or variable temperatures.
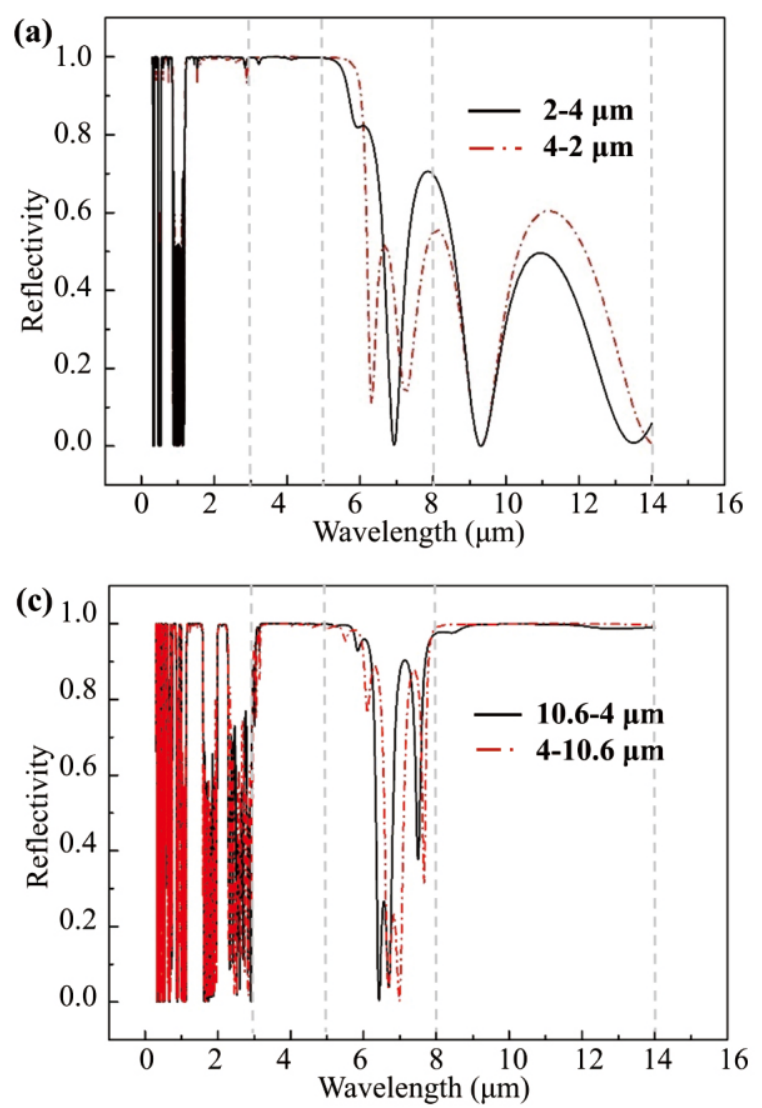

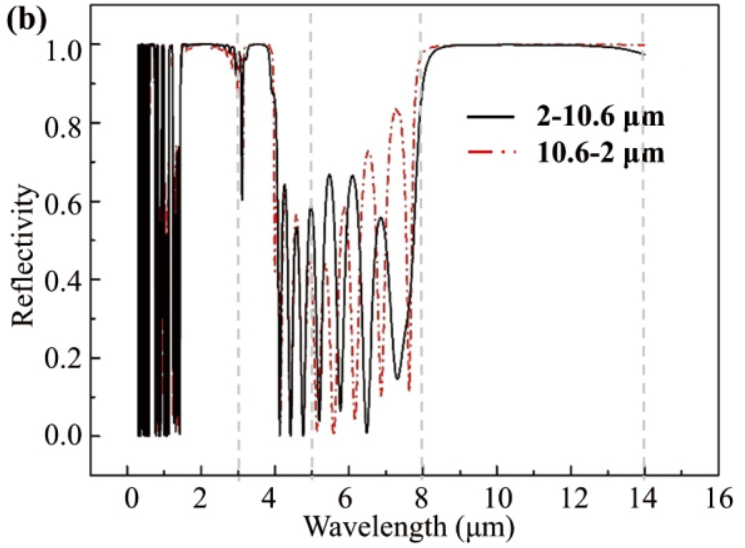

Fig. 5 Reflectivity of multilayer films with different stacking modes: (a) $\lambda_{0}=2 \mu \mathrm{m}$ and $\lambda_{0}=4 \mu \mathrm{m}$ films; (b) $\lambda_{0}$ $=2 \mu \mathrm{m}$ and $\lambda_{0}=10.6 \mu \mathrm{m}$ films; (c) $\lambda_{0}=4 \mu \mathrm{m}$ and $\lambda_{0}=$ $10.6 \mu \mathrm{m}$ films. 
However, when it comes to domestic or industrial environments, medium and low temperature are more common than extreme temperature areas. Hence, in the following sections, the mid-far infrared atmospheric window was mainly considered as radiation effect that corresponded to 4-10.6 $\mu \mathrm{m}$-central-wavelength stacked films structure.

\section{Period numbers}

In order to study the influence of the period numbers $N_{\mathrm{c}}$ on the reflection characteristics, the 4-10.6 $\mu \mathrm{m}$-central-wavelength stacked films was calculated, with $0.5-14 \mu \mathrm{m}$ wavelength range. Numerical simulation was carried out using the transfer matrix method, where the values of $N_{\mathrm{c}}$ were taken as $2,4,6$, and 8 , respectively. The results are shown in Fig. 7.

Increase of $N_{\mathrm{c}}$, resulted in the reflectance at the mid-far infrared atmospheric window band (3-5 $\mu \mathrm{m}$ and 8-14 $\mu \mathrm{m})$ to become closer to 1 .
This means the larger $N_{c}$, the better the radiation cooling effect of the multilayer films. When $N_{\mathrm{c}}=6$, the reflectance of the overall curve in the mid-far infrared band was above 0.99. This suggests that the structure accommodated the cooling radiation requirement in the medium temperature range of 580-966 $\mathrm{K}$, as well as the medium -low temperature range of 362-207 K. However, comparing Fig. 7 (c) and Fig. 7 (d), it can be seen when $N_{\mathrm{c}}>6$, the influence of increasing $N_{\mathrm{c}}$ was no longer affected the reflectivity obviously. Based on this trend, it can be inferred that the optimum radiation cooling effect can be obtained when the period number $N_{\mathrm{c}}=6$, which is of economic benefit. Hence, the period number $N_{\mathrm{c}}=6$ was used subsequent calculations.

\section{Refractive index ratio}

The refractive index ratio was analyzed to generate the its effects on the radiative cooling characteristics of the multilayer films, where the 4-
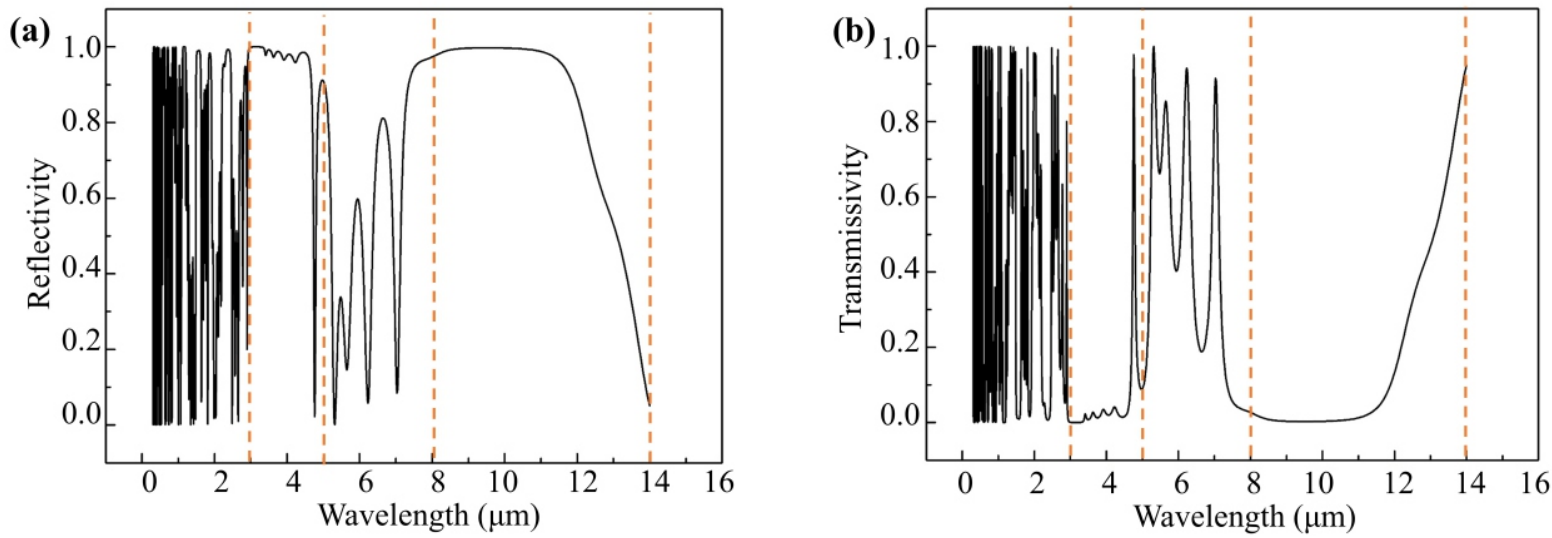

Fig. 6 (a) Reflectivity of multilayer films stacked at three central wavelengths; (b) transmissivity of multilayer films stacked at three central wavelengths.
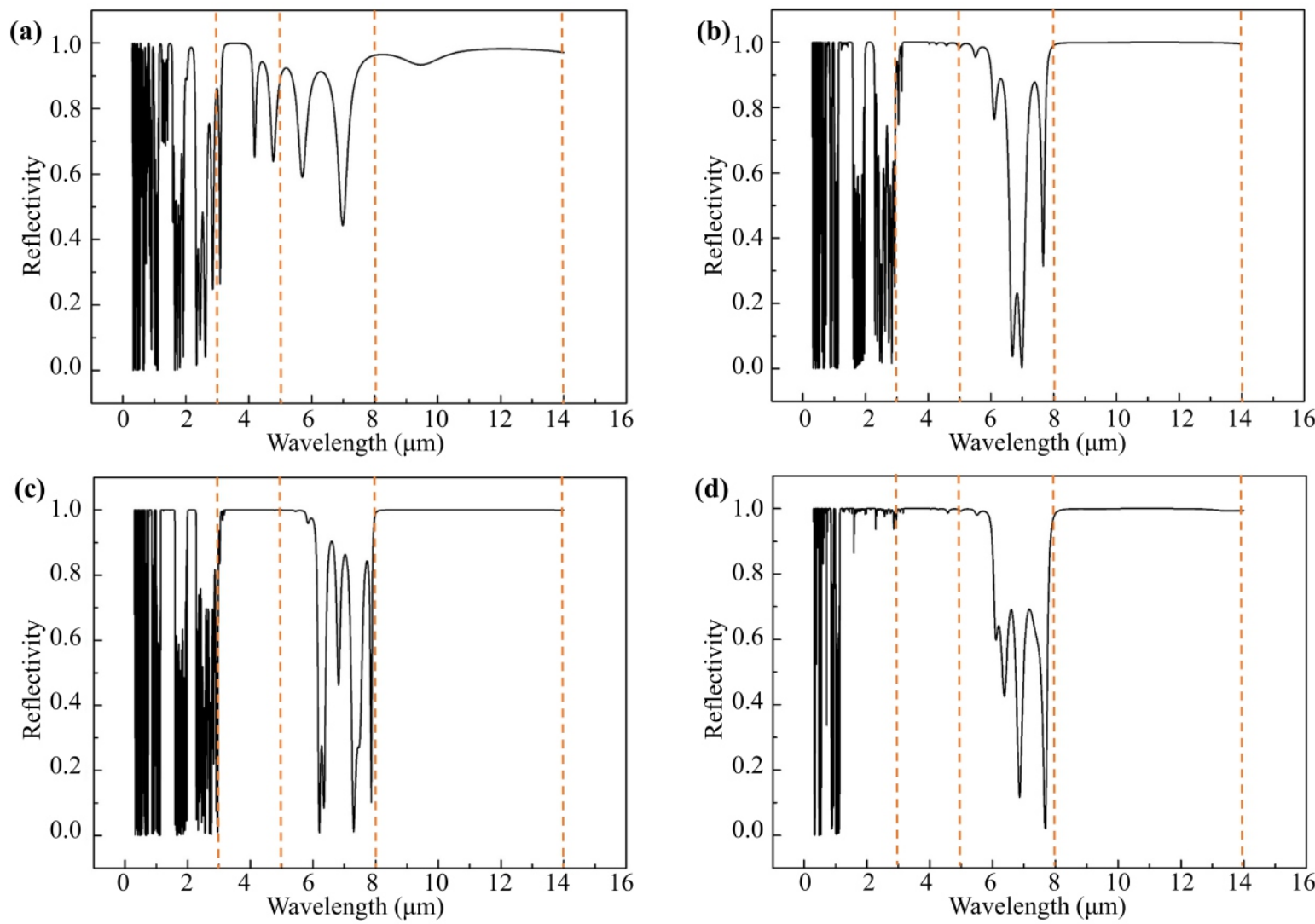

Fig. 7 Reflectivity of multilayer films with different period numbers: (a) $N_{\mathrm{c}}=2$; (b) $N_{\mathrm{c}}=4$; (c) $N_{\mathrm{c}}=6$; (d) $N_{\mathrm{c}}=8$. 
$10.6 \mu \mathrm{m}$-central-wavelength stacked films were calculated with 0.5-14 $\mu \mathrm{m}$ wavelength range. The low emissivity materials $\mathrm{PbS}, \mathrm{PbTe}$ and $\mathrm{InP}$ were selected as comparative high refractive index materials, with their refractive index are 4.1, 5.8 and 3.1, respectively. In these three sets of different high refractive index material models, $\mathrm{SiO}_{2}$ was kept as a low refractive index material. According to the EMT, equations (9) was used to design the radiative cooling films' structural parameters (Table 2). The higher the refractive index ratio, the thinner films structure became (Table 2). The results of the numerical calculation are shown in Fig. 8.

From Fig. 8, the reflectivity of the multilayer films for the mid-far infrared atmosphere window was $\mathrm{PbTe} / \mathrm{SiO}_{2}>\mathrm{PbS} / \mathrm{SiO}_{2}>\mathrm{InP} / \mathrm{SiO}_{2}$. That is, the greater the refractive index ratio, the better the radiation cooling effect of the structure. The $\mathrm{PbTe}$ material as the largest refractive index achieved a reflectance which was greater than 0.99 in both the middle and far infrared atmosphere windows. This phenomenon provided a guide for material design. The thinner the film, the less space it takes and the less material it consumes, whereas, the higher the processing required. Therefore, it is necessary to consider the application effect and economic benefits to select materials comprehensively.

\section{Surface roughness}

Limited by current manufacturing technology, ${ }^{41-44}$ film surfaces may not be completely smooth. Therefore, surface roughness was analyzed to obtain the effect on radiative cooling in section, where the 4-10.6 $\mu \mathrm{m}$ central-wavelength stacked films was calculated at $0.5-14 \mu \mathrm{m}$ wavelength. In addition, six root mean squared (RMS) roughness models were built based on Otsu's research, ${ }^{45}$ with the roughness values $\sigma_{\text {RMS }}$ were $0.007 \mu \mathrm{m}, 0.014 \mu \mathrm{m}, 0.022 \mu \mathrm{m}, 0.031 \mu \mathrm{m}, 0.056 \mu \mathrm{m}$ and $0.118 \mu \mathrm{m}$, respectively. These models took a random distribution of 1000 seeds, and the distribution of the models with different roughness was the same. According to equation (10) and equation (11), the $B R D F$ of the different radiative cooling models were simulated, whose results at the wavelength of $10.6 \mu \mathrm{m}$ are shown in Fig. 9.

The distribution of $B R D F$ is basically consistent with the distribution of the rough surfaces (Fig. 9). That is, at a temperature of $273 \mathrm{~K}$, as $\sigma_{\mathrm{RMS}}$ increased, the average value of $B R D F$ was unstable (initially increased, then decreased and increased). When the $\sigma_{\mathrm{RMS}}=$ $0.031 \mu \mathrm{m}$, the average value of $B R D F$ was 0.00056 , that is, the reflectivity was the lowest, and the radiation cooling effect at $273 \mathrm{~K}$ was the worst. When the $\sigma_{\mathrm{RMS}}=0.118 \mu \mathrm{m}$, the mean value of $B R D F$

Table 2 Film thickness corresponding to different materials with different central wavelengths.

\begin{tabular}{cccc}
\hline$\lambda_{0} / \mu \mathrm{m}$ & Material & Refractive index & Film thickness $/ \mu \mathrm{m}$ \\
\hline \multirow{4}{*}{$4 \mu \mathrm{m}$} & $\mathrm{PbTe}$ & 5.8 & 0.172 \\
& $\mathrm{PbS}$ & 4.1 & 0.244 \\
& $\mathrm{InP}$ & 3.1 & 0.323 \\
& $\mathrm{SiO}_{2}$ & 1.45 & 0.69 \\
\hline \multirow{3}{*}{$10.6 \mu \mathrm{m}$} & $\mathrm{PbTe}$ & 5.8 & 0.457 \\
& $\mathrm{PbS}$ & 4.1 & 0.646 \\
& $\mathrm{InP}$ & 3.1 & 0.855 \\
& $\mathrm{SiO}_{2}$ & 1.45 & 1.828 \\
\hline
\end{tabular}

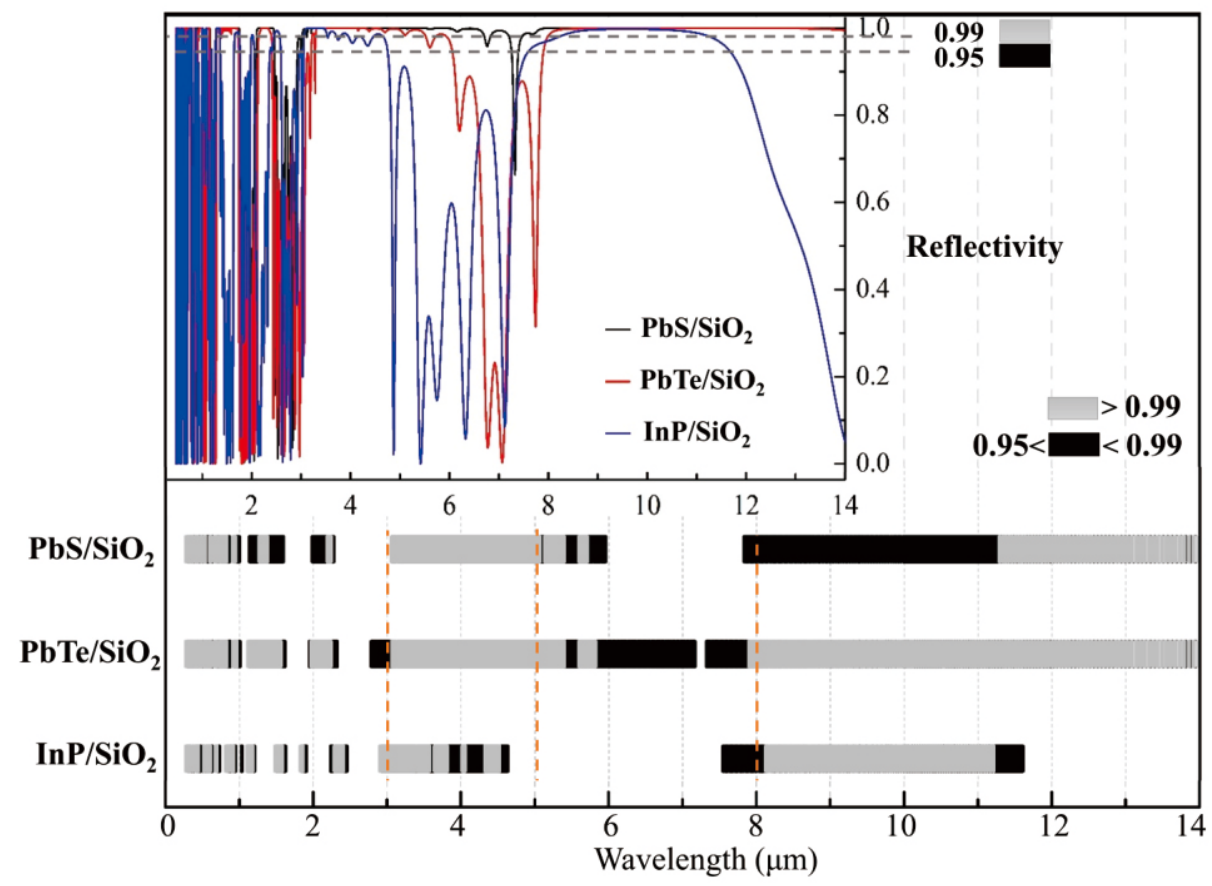

Fig. 8 The reflectivity of multilayer films with different materials. 
was 0.00317 , and the average effect of radiant cooling was the best. As the roughness increased, the difference between the maximum and minimum values of $B R D F$ was also increased. That is, the greater the roughness of multilayer films, the greater the volatility of the reflectance.

Overall, the roughness model of $\sigma_{\text {RMS }}=0.014 \mu \mathrm{m}$ achieved the most stable and better reflection. It can be inferred that to obtain a better radiation cooling effect in practical applications, it's necessary to comprehensively consider the processing cost and stability of surface roughness.

\section{Conclusions}

This present study examined the radiation characteristics of multilayer films in the band of 0.3-14 $\mu \mathrm{m}$ using transmission matrix method. Based on the effective medium theory, multilayer films at three atmospheric window bands were designed. For flexible use in different temperature ranges, the effect of stacking modes, period number, refractive index ratio, as well as the surface roughness, were considered in the multilayer films. These radiative cooling multilayer films can increase the reflectivity of energy at three atmospheric windows greater than 0.99 , with the overall reflectivity being higher than 0.95 . The larger the period number, the better the radiative cooling until it is greater than 6 where the heat insulation increase becomes obvious. The bottom layer was most affected by the overall contribution of this device. The ratio of the refractive index of the two materials was proportional to the effect

of the radiation heat insulation effect. Considering the influence of the actual surface roughness, the $0.014 \mu \mathrm{m}$ roughness model achieves the most stable and improve reflection performance. This research provided a new way to design and analyze the mechanism of radiative cooling multilayer films in high temperature and variable temperature environments, which can be widely used in existing processing technology.

\section{Conflict of interest}

There are no conflicts to declare.

\section{Acknowledgements}

The authors are grateful to National Natural Science Foundation of China (No.51536001 and No.51776050) for supporting this research.

\section{Nomenclature}

$\begin{array}{lll}d & \text { thickness } & \mu \mathrm{m} \\ E & \text { electric field intensity } & (\mathrm{A} / \mathrm{m}) \\ H & \text { magnetic field intensity } & (\mathrm{V} / \mathrm{m}) \\ k & \text { wave vector } & \mu \mathrm{m}^{-1} \\ n & \text { refractive index } & - \\ N_{\mathrm{c}} & \text { period numbers } & -\end{array}$

(a) $\sigma_{\mathrm{RMS}}=0.007 \mu \mathrm{m}$

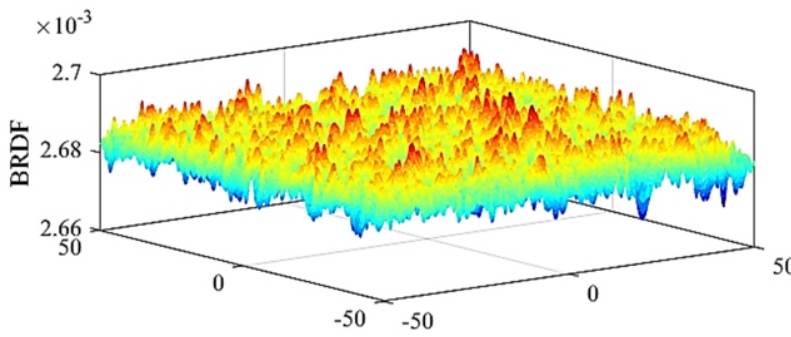

(c) $\sigma_{\mathrm{RMS}}=0.022 \mu \mathrm{m}$

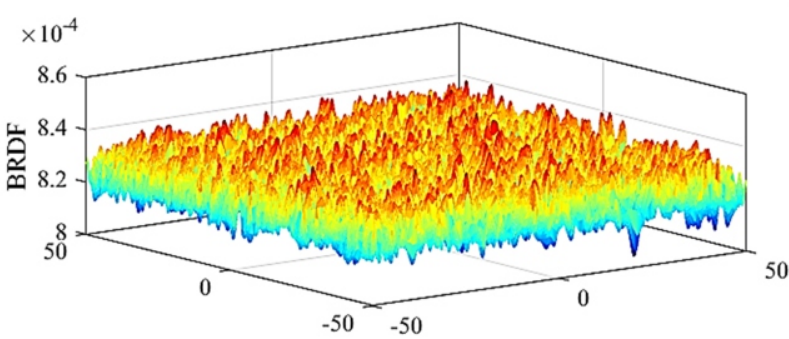

(b) $\sigma_{\mathrm{RMS}}=0.014 \mu \mathrm{m}$

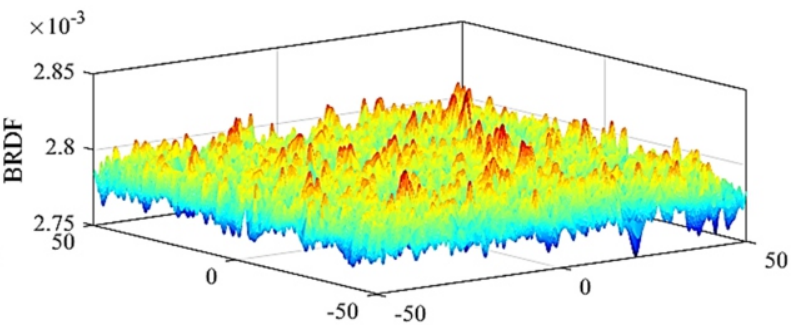

(d) $\sigma_{\mathrm{RMS}}=0.031 \mu \mathrm{m}$

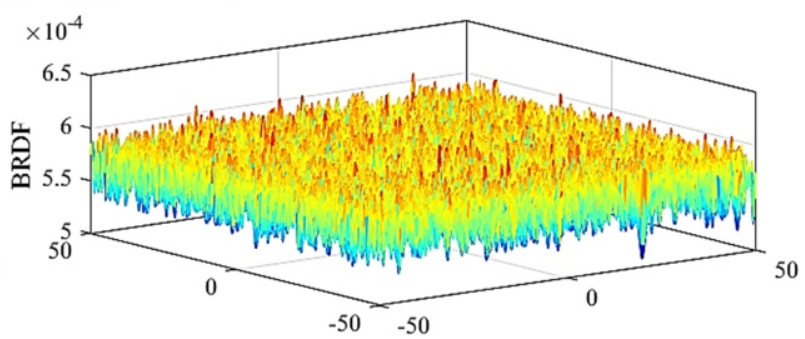

(f) $\sigma_{\mathrm{RMS}}=0.118 \mu \mathrm{m}$

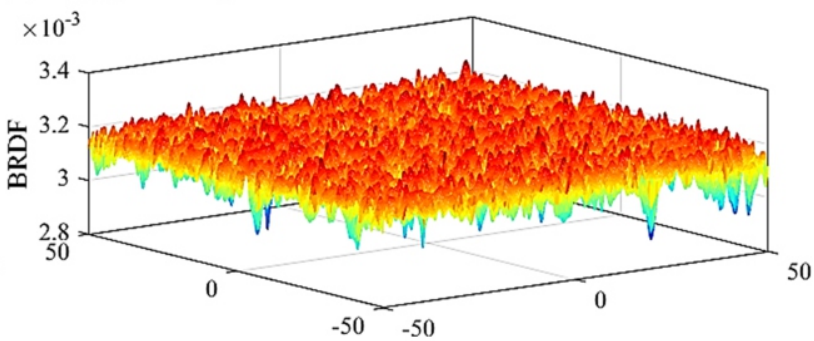

Fig. $9 \mathrm{BRDF}$ distribution with different roughness structures: (a) $\sigma_{\mathrm{RMS}}=0.007 \mu \mathrm{m}$; (b) $\sigma_{\mathrm{RMS}}=0.014 \mu \mathrm{m}$; (c) $\sigma_{\mathrm{RMS}}=0.022 \mu \mathrm{m}$; (d) $\sigma_{\mathrm{RMS}}=0.031 \mu \mathrm{m}$; (e) $\sigma_{\mathrm{RMS}}=0.056 \mu \mathrm{m} ;(\mathrm{f}) \sigma_{\mathrm{RMS}}=0.118 \mu \mathrm{m}$. 


$\begin{array}{lll}P & \text { the powder } & \text { W } \\ R & \text { reflectantivity } & - \\ r & \text { reflection coefficient } & - \\ T & \text { transmissivity } & - \\ t & \text { transmission coefficient } & - \\ x & \text { positive integer number } & \\ \delta & \text { displacement thickness } & \mu \mathrm{m} \\ \theta & \text { zenith angle } & - \\ \lambda & \text { the wavelength } & \mu \mathrm{m} \\ \sigma \mathrm{RMS} & \text { root mean squared roughness } & - \\ \varphi & \text { azimuth angle } & - \\ \text { Subscript } & \\ i & \text { layer number } & - \\ j & \text { incident wave direction } & -\end{array}$

\section{References}

1. J. Huang and $\mathrm{K}$. Yu, New nonlinear optical materials: theoretical research, Nova Publishers, New York, 2007.

2. S. Sklan and B. Li, Natl. Sci. Rev., 2018, 5, 138-141.

3. J. Xie and B.Cao, Comput. Fluids, 2018, 160, 42-50.

4. C. Qin, K. Kang, I. Lee and B. Lee, Sol. Energy, 2017, 150, 512-520.

5. G. Qiao, H. Cao, F. Jiang, X. She, L. Cong, Q. Liu, X. Lei, A. Alexiadis and Y. Ding, ES Energy Environ., 2019, 4, 48-58.

6. Y. Peng, J. Chen, A. Song, P. Catrysse, P. Hsu, L. Cai, B. Liu, Y. Zhu, G. Zhou, D.Wu and H. Lee, Nature Sustainability, 2018, 1, 105-112.

7. T. Li, Y .Zhai, S. He, W. G an, Z. Wei, M. Heidarinejad, D. Dalgo, R. Mi, X. Zhao, J. Song and J. Dai, Sci., 2019, 364,760-763.

8. S. Angaiah, S. Arunachalam, V. Murugadoss and G. Vijayakumar, ES Energy Environ., 2019, 4, 59-65.

9. C. Jagtap, V. Kadam, S. Jadkar and H. Pathan, ES Energy Environ., 2019, 3, 60-67.

10. M. Ferroukhi, K. Abahri, R. Belarbi, K. Limam and A. Nouviaire, Heat Mass Transfer, 2016, 52, 2257-2269.

11. B. Grabowska and J. Kasperski, J. Therm. Sci., 2018, 27, 463-469.

12. R. Mortimer, D. Rosseinsky and P. Monk, Electrochromic materials and devices. John Wiley \& Sons, New York, 2015.

13. B. Paul, Appl. Optics, 1984, 23, 370-372.

14. A. Gentle and G. Smith, Adv. Sci., 2015, 2, 1500119.

15. Y. Huang, M. Pu, Z. Zhao, X. Li, X. Ma and X. Luo, Opt. Commun., 2018, 407, 204-207.

16. B. Zhao, M.Hu, X. Ao, N. Chen and G. Pei , Appl. Energ., 2019, 236, 489513.

17. C. Granqvist and A. Hjortsberg, J. Appl. Phys., 1981, 52, 4205-4220.
18. M.Kecebas, M. Menguc, A. Kosar and K. Sendur, J. Quant. Spectrosc Radiat. Transfer, 2017, 198, 179-186.

19. L. Sun, K.Luo and J. Fan, Fuel, 2018, 231, 85-93.

20. S.Wang, A. Ameli, V. Shaayegan, Y. Kazemi, Y. Huang, H. Naguib and C. B. Park, Polymers, 2018, 3, 261,

21. T. Choy, Effective medium theory: principles and applications. Oxford University Press, Oxford, 2015.

22. P. Sheng, Introduction to wave scattering, localization and mesoscopic phenomena, Springer Science \& Business Media, Medford, 2006.

23. Z. Zhang, Nano/microscale heat transfer, McGraw-Hill, New York, 2007.

24. Z. Guo, ES Energy Environ., 2018, 1, 4-15.

25. H. Bao, J. Chen, X. K. Gu and B. Y. Cao, ES Energy Environ., 2018, 1, 16-55.

26. H. Wang, X. Liu, L. Wang and Z. Zhang, Int. J. Therm. Sci., 2013, 65, 62-69.

27. X. Liu, T. Bright and Z. Zhang, J. Heat Tran., 2014, 136, 092703.

28. X. Liu and Z. Zhang, ACS Photonics, 2015, 2, 1320-1326.

29. B. Zhao and Z. Zhang, J. Quant. Spectrosc. Radiat. Transfer, 2014, 135, 8189.

30. B. Zhao, B. Guizal, Z. Zhang, S. Fan and M. Antezza, Phys. Rev. B, 2017, 95, 245437.

31. R. Zhang and Z. Zhang, J. Quant. Spectrosc. Radiat. Transfer, 2017, 197, $132-140$.

32. X. Zhang and Y. Wu, Sci. Rep., 2015, 5, 7892.

33. R. Petersen, T. Pedersen, M. Gjerding and K. Thygesen, Phys. Rev. B, 2016, 94, 035128.

34. X. Huang, H. Yang, D. Wang, S. Yu, Y. Lou and L. Guo, J. Phys. D: Appl. Phys., 2016, 49, 325101.

35. X. Li, M. Wang and B. Zhang, J. Quant. Spectrosc. Radiat. Transfer, 2016, 179, $165-169$

36. F. G. Smith, J. S. Accetta and D. L. Shumaker, The Infrared \& ElectroOptical Systems Handbook. Atmospheric Propagation of Radiation, Volume 2, Infrared Information And Analysis Center Ann Arbor Mi, Fort Belvoir, 1993.

37. H. Schneider, T. Maier, J. Fleissner, M.Walther, P. Koidl, G. Weimann, W. Cabanski, M. Finck, P. Menger, W. Rode and J. Ziegler, Infrared Phys. Techn., 2005,47, 53-58.

38. J. Kim and M. Swaminathan, IEEE T. Adv. Packaging, 2002, 25, 189-199.

39 D. Smith, D. Vier, T. Koschny and C. Soukoulis, Phys. Rev. E, 2005, 71, 036617.

40. M. Modest, Radiative heat transfer. Academic press, Oakville, 2013.

41. H. Wang, Y. Kawahito, R. Yoshida, Y. Nakashima and K. Shiokawa, Int. J. Heat Mass Tran., 2018, 118, 562-569

42. H. Wang, Y. Kawahito, R. Yoshida, Y. Nakashima and K. Shiokawa, Opt. Lett., 2017, 42, 2251-2254

43. H. Wang, M. Nakanishi and Y. Kawahito, J. Mater. Process. Tech., 2017, 249, 193-201

44. R. Zhang, G.Chu, G.Vasilyev, P. Martin, A.Camposeo, L. Persano, D. Pisignano and E. Zussman, ACS Appl. Mater. Inter., 2019, 11, 19324-19330.

45. T. Otsu, K. Komatsu and S. Hashimura, Tribol. Int., 2019, 136, 1-12.

Publisher's Note Engineered Science Publisher remains neutral with regard to jurisdictional claims in published maps and institutional affiliations. 